\title{
An Automated Platform for Analysis of Phosphoproteomic Datasets: Application to Kidney Collecting Duct Phosphoproteins
}

\author{
Jason D. Hoffert ${ }^{\dagger} \ddagger$, Guanghui Wang $\ddagger$ $§$, Trairak Pisitkun ${ }^{\dagger}$, Rong-Fong Shen $§$, and Mark A. \\ Knepper ${ }^{*} \dagger$ \\ Laboratory of Kidney and Electrolyte Metabolism, Proteomics Core Facility ${ }^{\diamond}$, National Heart, Lung, \\ and Blood Institute, Bethesda, Maryland 20892
}

\begin{abstract}
Large-scale phosphoproteomic analysis employing liquid chromatography-tandem mass spectrometry (LC-MS/MS) often requires a significant amount of manual manipulation of phosphopeptide datasets in the postacquisition phase. To assist in this process, we have created software, PhosphoPIC (PhosphoPeptide Identification and Compilation), which can perform a variety of useful functions including automated selection and compilation of phosphopeptide identifications from multiple MS levels, estimation of dataset false discovery rate, and application of appropriate cross-correlation (XCorr) filters. In addition, the output files generated by this program are compatible with downstream phosphorylation site assignment using the Ascore algorithm, as well as phosphopeptide quantification via QUOIL. In this report, we utilized this software to analyze phosphoproteins from short-term vasopressin-treated rat kidney inner medullary collecting duct (IMCD). A total of 925 phosphopeptides representing 173 unique proteins were identified from membrane-enriched fractions of IMCD with a false discovery rate of $1.5 \%$. Of these proteins, 106 were found only in the membrane-enriched fraction of IMCD cells and not in whole IMCD cell lysates. These identifications included a number of well-studied ion and solute transporters including ClC-1, LAT4, MCT2, NBC3, and NHE1, all of which contained novel phosphorylation sites. Using a label-free quantification approach, we identified phosphoproteins that changed in abundance with vasopressin exposure including aquaporin-2 (AQP2), Hnrpa3, $\mathrm{IP}_{3}$ receptor 3, and pur-beta.
\end{abstract}

\section{Keywords}

phosphoproteomics; neutral loss; target decoy; LC-MS/MS; collecting duct; IMCD; mass spectrometry; label free; PhosphoPIC; proteomics

\footnotetext{
* To whom correspondence should be addressed. Mark A. Knepper, M.D. Ph.D., NIH Bldg. 10, Room 6N260, 10 CENTER DR MSC 1603, bethesda, MD 20892-1603;Phone,(301)496-3064;Fax,(301)402-1443;E-mail, knep@ helix.nih.gov.

tLaboratory of Kidney and Electrolyte Metabolism.

These authors contibuted equally to this manuscript.

\$Proteomics Core Facility.

Supporting Information Available: $\mathrm{MS}^{2}$ summary and multiconsensus files (Supporting Information Table 1); $\mathrm{MS}^{3}$ summary and multiconsensus files (Supporting Information Table 2); combined $\mathrm{MS}^{2}$ and $\mathrm{MS}^{3}$ datasets with Ascore results (Supporting Information Table 3); summary of newly identified IMCD phosphoproteins including gene names, accession numbers, site(s) of phosphorylation, and relevant GO terms (Supporting Information Table 4); relevant statistics for phosphoproteins that changed in abundance with shortterm vasopressin treatment (Supporting Information Table 5); instructional files for using PhosphoPIC (readme_phosphoPIC.txt) and QUOIL (readme_QUOIL.txt). This material is available free of charge via the Internet at http://pubs.acs.org.
} 


\section{Introduction}

Large-scale "shotgun" LC-MS/MS analysis is a powerful method that can be used to identify and quantify phosphopeptides from a complex protein sample. ${ }^{1-5}$ This method usually involves analysis of a mixture of phosphopeptides obtained from either whole cell lysates or complex subcellular fractions. With the current availability of high-resolution and highsensitivity mass spectrometers, MS datasets generated by this particular phosphoproteomic approach have grown progressively larger and more cumbersome. In addition, there are a number of attributes unique to phosphopeptide datasets that create additional challenges in the postacquisition phase.

One distinctive characteristic of phosphopeptide datasets is that they often include both phosphorylated and nonphosphorylated peptides. Even with the addition of initial chromatography steps such as strong cation exchange and immobilized metal affinity chromatography (IMAC) to enrich for phosphopeptides, a significant number of nonphosphorylated peptides will remain in the sample during MS analysis. Removing nonphosphorylated peptide identifications in silico is necessary for both accurate estimation of dataset false discovery rate (FDR) and appropriate application of spectral quality filters (i.e., XCorr, RSp). The presence of a relatively high number of "single peptide" identifications in phosphopeptide datasets heightens the need for appropriate filtering and accurate FDR estimation.

The inclusion of phosphopeptide data from multiple MS levels (i.e., $\mathrm{MS}^{2}$ and $\mathrm{MS}^{3}$ ) also complicates postacquisition analysis. Higher quality $\mathrm{MS}^{3}$ spectra are utilized to confirm poorer quality $\mathrm{MS}^{2}$ identifications, ${ }^{6}$ and $\mathrm{MS}^{3}$ data often contain a significant number of unique phosphopeptides not found at the $\mathrm{MS}^{2}$ level. However, the presence of the same phosphopeptide in both MS2 and MS3 scans can lead to redundant analysis if these files are analyzed separately. To simplify this process, both $\mathrm{MS}^{2}$ and $\mathrm{MS}^{3}$ datasets should be analyzed as a single integrated file.

While these data manipulations can be performed manually, the process is both labor-intensive and prone to user error. Programs that automate particular aspects of phosphoproteomic data analysis are currently available. ${ }^{2,3,7,8}$ However, we were especially interested in creating a more general workflow that suited our needs in the analysis of phosphopeptide identifications generated by the SEQUEST search algorithm.

To create a more integrated, user-friendly platform, we developed PhosphoPIC, a Windowsbased software suite written in Visual C++ that utilizes SEQUEST search results generated from multiple MS levels to create a single filtered phosphopeptide dataset. This software provides an intuitive linear workflow by including individual programs for most steps of postacquisition analysis: phosphopeptide selection, FDR estimation, spectral quality filtering, and compilation of $\mathrm{MS}^{2}$ and $\mathrm{MS}^{3}$ search results. In this study, PhosphoPIC was successfully used to filter and compile phosphopeptide datasets generated from an LC-MS/MS shotgun analysis of membrane-enriched samples of renal inner medullary collecting duct (IMCD). The software was also useful in generating compatible files for automated phosphorylation site assignment and label-free phosphopeptide quantification.

\section{Materials and Methods}

\section{Sample Preparation}

IMCD suspensions were prepared from rat kidney inner medullas using the method of Stokes et al. ${ }^{9}$ with modifications. ${ }^{10}$ After isolation, IMCD suspensions were incubated for $10 \mathrm{~min}$ in the absence or presence of $10 \mathrm{nM}$ [deamino-Cys, ${ }^{1} \mathrm{D}-\mathrm{Arg}^{8}$ ] vasopressin (dDAVP), a V2- 
receptor-specific analog of the anti-diuretic hormone, arginine vasopressin. After centrifugation at $10000 \times \mathrm{g}$ for $30 \mathrm{~s}$, pelleted IMCD tubules were sonicated in ice-cold homogenization buffer: $250 \mathrm{mM}$ sucrose, $10 \mathrm{mM}$ triethanolamine, $1 \times$ Complete protease inhibitor cocktail (Roche, Mannheim, Germany), $1 \times$ HALT phosphatase inhibitor (Pierce, Rockford, IL), pH adjusted to 7.6. Samples were spun at $1000 \times \mathrm{g}$ for $10 \mathrm{~min}$ to pellet unbroken cells and debris. The supernatant from this step was centrifuged at $200000 \times \mathrm{g}$ for $1 \mathrm{~h}$. This "membrane-enriched" pellet (200-600 $\mu \mathrm{g}$ protein) was resuspended in $6 \mathrm{M}$ guanidine-HCl/50 $\mathrm{mM}$ ammonium bicarbonate followed by reduction, alkylation, trypsin digestion, and desalting as previously described. ${ }^{4}$ In this study, no detergents were used to solubilize membrane protein samples since their presence did not increase the yield of identified phosphopeptides (data not shown). To enrich for phosphopeptides, desalted samples in $0.1 \%$ formic acid were subjected to IMAC (Pierce, Phosphopeptide Isolation Kit) using the supplied protocol with slight modifications. ${ }^{4}$ Samples were then desalted using C18 Ziptips (Millipore) prior to analysis by mass spectrometry.

\section{LC-MS/MS Analysis}

Samples were analyzed on an Agilent 1100 nanoflow LC system (Palo Alto, CA) connected to a Finnigan LTQ FT mass spectrometer (Thermo, San Jose, CA) as previously described. ${ }^{4}$ Briefly, the peptide mixture injected by the autosampler was loaded onto a $\mathrm{C} 18$ precolumn (Agilent) for desalting. Captured peptides were directed to a PicoFrit reversed-phase analytical column (New Objective, Woburn, MA) and eluted by using a linear gradient of acetonitrile $(0-60 \%$ in $45 \mathrm{~min})$ in $0.1 \%$ formic acid at a flow rate of $250 \mathrm{~nL} / \mathrm{min}$. Eluted peptides were ionized and analyzed using the LTQ-FT mass spectrometer equipped with a nanospray ion source. Spectra were recorded in data-dependent acquisition mode and the dynamic exclusion option was enabled. Survey MS scans were acquired by either the LTQ (linear ion trap) or the FT-ICR (Fourier transform-ion cyclotron resonance) detector (with a resolution of 100000 at $\mathrm{m} / \mathrm{z}$ of 400), and each survey MS scan was followed by five MS/MS scans using the LTQ. MS $^{3}$ scans were triggered if a neutral-loss fragment (loss of 98, 49, or 32.7 from the precursor ion) was observed in the corresponding MS/MS scans.

\section{Target-Decoy Database Search}

MS/MS spectra were searched using BioWorks 3.1 (Thermo Scientific) running the SEQUEST algorithm on a concatenated database containing both the forward (target) and reversed complement (decoy) of the most current version of the Rat Refseq Database (National Center for Biotechnology Information). The following parameters were used: 2 missed trypsin cleavage sites permitted, fixed modification of 57.0214 on cysteine, variable modifications of 15.9949 on methionine and 79.9663 on serine, threonine, and tyrosine. For $\mathrm{MS}^{3}$ spectra, an additional variable modification of -18.0106 on serine and threonine was added to account for dehydration of these two amino acids due to neutral loss of phosphoric acid.

\section{Phosphopeptide Analysis using PhosphoPIC}

PhosphoPIC was created with Visual C++ as a set of Microsoft Windows-based programs with output files generated in a flexible, tab-delimited text format. (This software is freely available for noncommercial purposes upon request.) Through the "Phosphopeptide Selection/Filtering" module of PhosphoPIC, all.dta and.out files contained in the SEQUEST search results directory were used to generate a filtered dataset which was constrained by various parameters including the presence of at least one phosphorylated amino acid, a maximum preliminary score rank $(\mathrm{RSp})=1$, and a minimum deltaCn $=0$. Concomitantly, the PhosphoPIC software automatically adjusted the minimum cross-correlation (XCorr) filter for each individual charge state $(+1,+2$, +3 ) separately in order to optimally meet predetermined target false discovery rates (FDRs) based on the number of allowable random matches from the reversed "decoy" database. The 
actual FDR was calculated as $2 \mathrm{R} /(\mathrm{F}+\mathrm{R})$ and the adjusted FDR was calculated as $\mathrm{R} / \mathrm{F}$, where $\mathrm{R}$ is the number of reversed hits and $\mathrm{F}$ is the number of forward hits. Setting the maximum RSp value as low as possible allowed PhosphoPIC to choose lower XCorr cutoff values, resulting in a higher number of identifications for a given target FDR. However, filtering for a maximum $\mathrm{RSp}=1$ excludes possible "true positive" identifications that were "first matches" (highest XCorr value) yet did not have the highest preliminary scores. Thus, there is an obvious tradeoff between coverage and quality when filtering phosphopeptide datasets. The Phosphopeptide Selection/Filtering program was designed for general purpose filtering of peptide identifications at specified target FDRs. Thus, it is also compatible with LC-MS/MS analysis of non-phosphorylated peptides, although not utilized in this capacity in this study.

The end result of this initial filtering process was the creation of two separate spreadsheet files, a multiconsensus report similar to ones obtained through Bioworks, as well as a summary file containing useful information such as XCorr filters applied, actual FDR, adjusted FDR (calculated after removal of random matches), total redundant and nonredundant peptide count for both forward and reversed databases, and number of proteins identified for a given peptide count. $\mathrm{MS}^{2}$ and $\mathrm{MS}^{3}$ datasets filtered with a target $\mathrm{FDR}=2 \%$ were used for all subsequent phosphopeptide analysis. The optimal XCorr cutoff values established for this FDR were 2.02 $(+1), 2.92(+2)$, and $4.40(+3)$ for MS; ${ }^{2} 1.58(+1), 2.87(+2)$, and $3.23(+3)$ for MS. ${ }^{3}$ Adjusted FDRs for $\mathrm{MS}^{2}$ and $\mathrm{MS}^{3}$ datasets were 0.67 and $0.81 \%$, respectively.

After phosphopeptide selection and filtering, datasets from both MS levels were merged using the "MSn Compilation" module of PhosphoPIC, which generated another pair of only). These files were used to determine which phosphopeptides were identified at each MS level.

Phosphopeptides from $\mathrm{MS}^{2}$ spectra were selected using PhosphoPIC and the corresponding.dta and.out files for those identifications were analyzed using Ascore

(http://ascore.med.harvard.edu/ascore.php), a statistical algorithm that measures the probability of correct phosphorylation site localization based on the presence and intensity of site-determining ions in $\mathrm{MS}^{2}$ spectra. ${ }^{1}$ Phosphorylation sites with an Ascore $\geq 19$

(corresponding to $>99 \%$ certainty) were considered unambiguously assigned. Since the Ascore algorithm is only compatible with $\mathrm{MS}^{2}$ spectra, phosphorylation sites from phosphopeptides found only at the $\mathrm{MS}^{3}$ level were assigned by manual examination of the spectra.

\section{Label-Free Quantification}

Label-free phosphopeptide quantification was performed using the QUOIL (quantification without isotopic labeling) program ${ }^{11}$ which can accept summary files generated by PhosphoPIC for individual sample trials. QUOIL quantifies reconstructed peak ion chromatograms from separate LC-MS/MS runs and has been used successfully for quantification of both non-phosphoryated ${ }^{11}$ and phosphorylated peptides. ${ }^{4}$ To ensure accurate quantification, MS data were collected using the high-resolution FT-ICR analyzer for precursor ion scanning while the LTQ was used for analysis of fragment ion spectra. The following parameters were used for label-free quantification of MS data: maximum $\mathrm{RSp}=2$, minimum deltaCn $=0$, target FDR $=2 \%$, precursor $m / z=$ theoretical $\left(\right.$ for MS ${ }^{2}$ ), measured (for $\mathrm{MS}^{3}$ ); peptide ratio calculated using peak area; peptide ratio normalization by median at raw file level; protein ratio calculated using median; precursor $\mathrm{m} / \mathrm{z}$ tolerance $=0.05 \mathrm{Da}$; peak window $=1$ min; minimal $\mathrm{S} / \mathrm{N}=1$; maximal $\mathrm{FWHM}=0.3 \mathrm{~min}$; min ratio change: 0.25 ; peptide set $=$ union, peak RT tolerance between samples $=3 \mathrm{~min}$. 


\section{Results and Discussion}

\section{General Strategy for Isolation and Analysis of Phosphopeptides}

Rat inner medullary collecting duct (IMCD) tubules were enriched from whole inner medulla by a combination of collagenase digestion and differential centrifugation as detailed in the "Materials and Methods" section. Following short-term vasopressin treatment (10 nM dDAVP for $10 \mathrm{~min}$ ), samples were processed as shown in Figure 1A. Cells were disrupted by sonication, followed by differential centrifugation to produce a "membrane-enriched" $200000 \mathrm{~g}$ pellet. This pellet was resuspended in guanidine and digested with trypsin. Phosphopeptides were enriched from total peptide digests by using IMAC prior to LC-MS/MS analysis.

Postacquisition analysis was performed according to the scheme presented in Figure 1B. Both $\mathrm{MS}^{2}$ and $\mathrm{MS}^{3}$ spectra were searched using the target-decoy approach,, 12 which employed a concatenated database that included both forward and reversed sequences of the latest version of the rat Refseq database. The target-decoy approach is the prevailing method for estimating false discovery rates of MS datasets and is based on the principle that incorrect spectral matches have an equal probability of occurring in either forward ("target") or reversed ("decoy") databases. MS search results were then analyzed using PhosphoPIC. As described more thoroughly in "Materials and Methods", these programs perform 3 main functions: phosphopeptide selection/filtering, MSn compilation, and.dta/.out file selection. The first two functions help to organize, filter, and summarize phosphopeptide identifications, whereas the third function is used to generate the input file for automated phosphorylation site assignment. A more detailed workflow for the Phosphopeptide Selection/Filtering and MSn Compilation modules of PhosphoPIC is given in Figure 1C and D, respectively.

\section{Phosphopeptide Selection/Filtering}

To begin the Phospho-PIC analysis, MS datasets were filtered to remove non-phosphorylated peptide identifications. Appropriate XCorr filters were also applied, iteratively, based on userdefined "target FDR" values from 1 to 10\%. As shown in Figure 2, as the target FDR was increased, the number of phosphopeptide identifications from both the forward and reversed database increased due to progressively lower XCorr cutoff values. When compared with $\mathrm{MS}^{2}$ phosphopeptide totals, the $\mathrm{MS}^{3}$ dataset consistently included a higher number of identifications for all corresponding target FDRs, highlighting the importance of analyzing data from multiple MS levels. The "actual FDR" takes into account an equal number of known and predicted random hits from the reversed and forward databases, respectively. The "adjusted FDR" excludes the random matches from the reversed database and reflects the smallest FDR value that can be estimated by the target-decoy approach. For subsequent analysis, we selected $\mathrm{MS}^{2}$ and $\mathrm{MS}^{3}$ phosphopeptide datasets that were filtered to produce a target $\mathrm{FDR}=2 \%$, which corresponded to adjusted FDRs $<1 \%$. Single files containing both summary and multiconsensus files are available for $\mathrm{MS}^{2}$ (Supporting Information Table 1) and $\mathrm{MS}^{3}$ (Supporting Information Table 2) datasets.

\section{MSn Compilation}

Following initial XCorr filtering and FDR estimation, $\mathrm{MS}^{2}$ and $\mathrm{MS}^{3}$ datasets were combined into a single output file (Supporting Information Table 3). A total of 925 redundant (268 nonredundant) phosphopeptides were identified, including only 7 false positive matches $(1.5 \%$ actual FDR) (Table 1). After removing false positives, 173 unique phosphoproteins remained. The majority of these proteins (126) were identified from single peptide matches, a common characteristic of phosphopeptide datasets. The fact that most phosphoproteins were identified only at the $\mathrm{MS}^{3}$ level (101) is in contrast to results from a recent large-scale phosphoproteomic analysis of nocodazole-arrested HeLa cell lysates. ${ }^{1}$ In that study, Beausoleil et al. reported that only $8.7 \%$ of all nonredundant phosphorylation sites were contributed solely by $\mathrm{MS}^{3}$ spectra. 
The reason for this discrepancy in the relative contribution of $\mathrm{MS}^{3}$ spectra to an entire phosphopeptide dataset is not yet known.

Of the 173 phosphoproteins identified from membrane-enriched IMCD samples, 106 of these proteins were not found in the current Collecting Duct Phosphoprotein Database (CDPD) (http://dir.nhlbi.nih.gov/papers/lkem/cdpd/), which contains only phosphoproteomic data from total IMCD cell lysates. ${ }^{4}$ Newly identified integral membrane phosphoproteins included a number of well-known collecting duct ion and solute transport proteins ClC-1, LAT4, MCT2, NBC3, and NHE1 (Table 2). The possibility that some of these new identifications resulted simply from run-to-run LC-MS/MS variability cannot be excluded. A functional classification of all 106 proteins identified from membrane-enriched IMCD is presented as a set of three heat maps based on established Gene Ontology (GO) terms (Figure 3), one for each major GO category (cellular component, biological process, and molecular function). The intensity values correspond to the number of distinct proteins associated with a particular GO term. As expected, GO terms relating to membrane localization (e.g., basolateral plasma membrane, integral to plasma membrane, membrane, membrane fraction) as well as membrane transport (e.g., ion transport, sodium ion transport, transport, symporter activity, transporter activity) were more prominent in the membrane-enriched ("200k") fraction when compared to total IMCD cell lysates. The membrane-enriched fraction also included a large number of nuclear, ER, and mitochondrial proteins, most likely due to the nature of the homogenization and the lack of multiple lower speed centrifugation steps. A summary of all identified phosphoproteins including gene names, accession numbers, and relevant ontology data is included in Supporting Information Table 4.

\section{Phosphorylation Site Assignment}

To distinguish the correct site(s) of phosphorylation for each phosphopeptide, automated site assignment was performed on $\mathrm{MS}^{2}$ data using the Ascore algorithm. ${ }^{1}$ The Ascore value for each phosphopeptide is included in Supporting Information Table 3. In all, 59 distinct phosphorylation sites were unambiguously assigned (Ascore $\geq 19$ ). For the most part, these sites were identical to those found in corresponding $\mathrm{MS}^{3}$ spectra of the same peptide. Manual spectral validation was performed on phosphopeptides found only at the $\mathrm{MS}^{3}$ level. Of the 140 nonredundant phosphorylation sites among the 106 phosphoproteins unique to the membraneenriched fraction of rat IMCD, 41 had been previously identified and 76 were novel according to the online phosphoprotein database PhosphoSite (www.phosphosite.org). (PhosphoSite includes phosphorylation site information mostly for human and mouse proteins. If a site corresponding to the rat sequence was identified in mouse and/or human, then this site was considered "previously identified" even if it had not been identified in rat.) The remaining 23 sites could not be definitively assigned, although the corresponding phosphopeptides were still useful for phosphoprotein identification. For $\mathrm{MS}^{2}$ spectra, Ascore values $<19$ were considered ambiguous. For $\mathrm{MS}^{3}$ spectra, an ambiguous assignment resulted from unsuccessful manual inspection. When residues could not be definitively assigned, lack of assignment was usually due to a lack of distinguishing ion peaks surrounding the potential residue(s) or to generally poor spectral quality. A complete list of all successfully assigned and ambiguous phosphorylation sites is included in Supporting Information Table 4.

\section{Phosphopeptide Quantification}

To identify phosphopeptides that changed in abundance due to vasopressin treatment, labelfree quantification was performed on IMCD samples incubated with vehicle control $(n=3)$ and IMCD samples incubated with $10 \mathrm{nM}$ dDAVP for $10 \mathrm{~min}(n=3)$. Initially, PhosphoPIC was used to generate a separate $\mathrm{MS}^{2}$ multiconsensus file for each sample. These files were then submitted to QUOIL to allow reconstruction of the corresponding parent ion chromatograms. The entire process was then repeated for $\mathrm{MS}^{3}$ data. While the majority of 
phosphopeptides did not change in abundance in the presence of vasopressin, a small number of phosphopeptides showed significant changes including peptides for heterogeneous nuclear ribonucleoprotein $\mathrm{A} 3$ (decreased 4.0-fold), $\mathrm{IP}_{3}$ receptor 3 (decreased 5.8-fold), pur-beta (decreased 1.5-fold), and aquaporin-2 (increased 20.7-fold) (Supporting Information Table 5). Of particular relevance to our studies is the decrease in abundance of phosphorylated $\mathrm{IP}_{3}$ receptor 3. Although the sites of phosphorylation could not be unambiguously assigned, the identified peptide includes Ser-1832, a known target of protein kinase A (PKA), ${ }^{13}, 14$ which could potentially play a role in vasopressin signaling by modulating the release of intracellular $\mathrm{Ca}^{+2}$ from $\mathrm{IP}_{3}$-sensitive stores.

Another interesting phosphoprotein that was quantified in this study was aquaporin-2 (AQP2), the vasopressin-sensitive water channel. 15,16 Quantification of the AQP2 phosphopeptide ion chromatograms is shown in Figure 4. This particular AQP2 peptide was identified from an $\mathrm{MS}^{3}$ spectrum and contained three unambiguously assigned phosphorylation sites: Ser-256, Ser-261, and Ser-264. A previous phosphoproteomic study by our group included MS-based quantification of AQP2 at Ser-256 and Ser-261; ${ }^{4}$ however, none of the phosphopeptides in that study included phosphorylation at Ser-264. The dramatic increase in abundance of this phosphopeptide in vasopressin-treated samples is consistent with increased phosphorylation of AQP2 at Ser-256 in response to vasopressin. ${ }^{4,17,18}$ Although QUOIL has been routinely utilized to reconstruct parent ion chromatograms based on $\mathrm{MS}^{2}$-level peptide identifications, 4,11 these current results demonstrate the ability of QUOIL to measure relative abundance ratios of phosphopeptides identified from $\mathrm{MS}^{3}$ spectra as well.

\section{Conclusions}

In this paper, we have introduced a new software application, PhosphoPIC, for analysis of phosphopeptides from large-scale LC-MS/MS datasets and have utilized this software to successfully analyze phosphopeptides from a membrane-enriched fraction of rat IMCD. This software was intuitive to use and powerful in its ability to organize, filter, and compile large phosphopeptide datasets from multiple MS levels. A major advantage of using PhosphoPIC is its ability to interface with other programs for analyzing phosphoproteomic data such as QUOIL and Ascore, thus creating an automated platform that encompasses most aspects of phosphopeptide analysis.

\section{Supplementary Material}

Refer to Web version on PubMed Central for supplementary material.

\section{Acknowledgements}

We thank Dr. Sean Beausoleil for assistance with Ascore analysis. This work was supported by the National Heart, Lung, and Blood Institute intramural budget ZO1-HL001285.

\section{References}

1. Beausoleil SA, Villen J, Gerber SA, Rush J, Gygi SP. A probability-based approach for high-throughput protein phosphorylation analysis and site localization. Nat Biotechnol 2006;24(10):1285-1292. [PubMed: 16964243]

2. Olsen JV, Blagoev B, Gnad F, Macek B, Kumar C, Mortensen P, Mann M. Global, in vivo, and sitespecific phosphorylation dynamics in signaling networks. Cell 2006;127(3):635-648. [PubMed: 17081983]

3. Lu B, Ruse C, Xu T, Park SK, Iii JY. Automatic validation of phosphopeptide identifications from tandem mass spectra. Anal Chem 2007;79(4):1301-1310. [PubMed: 17297928] 
4. Hoffert JD, Pisitkun T, Wang G, Shen RF, Knepper MA. Quantitative phosphoproteomics of vasopressin-sensitive renal cells: regulation of aquaporin-2 phosphorylation at two sites. Proc Natl Acad Sci USA 2006;103(18):7159-7164. [PubMed: 16641100]

5. Gruhler A, Olsen JV, Mohammed S, Mortensen P, Faergeman NJ, Mann M, Jensen ON. Quantitative phosphoproteomics applied to the yeast pheromone signaling pathway. Mol Cell Proteomics 2005;4 (3):310-327. [PubMed: 15665377]

6. Olsen JV, Mann M. Improved peptide identification in proteomics by two consecutive stages of mass spectrometric fragmentation. Proc Natl Acad Sci USA 2004;101(37):13417-13422. [PubMed: 15347803]

7. Tao WA, Wollscheid B, O’Brien R, Eng JK, Li XJ, Bodenmiller B, Watts JD, Hood L, Aebersold R. Quantitative phosphoproteome analysis using a dendrimer conjugation chemistry and tandem mass spectrometry. Nat Methods 2005;2(8):591-598. [PubMed: 16094384]

8. Tabb DL, McDonald WH, Yates JR III. DTASelect Contrast: tools for assembling comparing protein identifications from shotgun proteomics. J Proteome Res 2002;1(1):21-26. [PubMed: 12643522]

9. Stokes JB, Grupp C, Kinne RK. Purification of rat papillary collecting duct cells: functional and metabolic assessment. Am J Physiol 1987;253(2 Pt 2):F251-F262. [PubMed: 3303974]

10. Pisitkun T, Bieniek J, Tchapyjnikov D, Wang G, Wu WW, Shen RF, Knepper MA. High-throughput identification of IMCD proteins using LC-MS/MS. Physiol Genomics 2006;25(2):263-276. [PubMed: 16449382]

11. Wang G, Wu WW, Zeng W, Chou CL, Shen RF. Label-free protein quantification using LC-coupled ion trap or FT mass spectrometry: Reproducibility, linearity, and application with complex proteomes. J Proteome Res 2006;5(5):1214-1223. [PubMed: 16674111]

12. Elias JE, Gibbons FD, King OD, Roth FP, Gygi SP. Intensity-based protein identification by machine learning from a library of tandem mass spectra. Nat Biotechnol 2004;22(2):214-219. [PubMed: 14730315]

13. Soulsby MD, Wojcikiewicz RJ. Calcium mobilization via type III inositol 1,4,5-trisphosphate receptors is not altered by PKA-mediated phosphorylation of serines 916, 934, and 1832. Cell Calcium. 2007

14. Soulsby MD, Wojcikiewicz RJ. The type III inositol 1,4,5-trisphosphate receptor is phosphorylated by cAMP-dependent protein kinase at three sites. Biochem J 2005;392(Pt 3):493-497. [PubMed: 16107208]

15. Nielsen S, Chou CL, Marples D, Christensen EI, Kishore BK, Knepper MA. Vasopressin increases water permeability of kidney collecting duct by inducing translocation of aquaporin-CD water channels to plasma membrane. Proc Natl Acad Sci USA 1995;92(4):1013-1017. [PubMed: 7532304]

16. Nielsen S, DiGiovanni SR, Christensen EI, Knepper MA, Harris HW. Cellular and subcellular immunolocalization of vasopressin-regulated water channel in rat kidney. Proc Natl Acad Sci USA 1993;90(24):11663-11667. [PubMed: 8265605]

17. Kuwahara M, Fushimi K, Terada Y, Bai L, Marumo F, Sasaki S. cAMP-dependent phosphorylation stimulates water permeability of aquaporin-collecting duct water channel protein expressed in Xenopus oocytes. J Biol Chem 1995;270(18):10384-10387. [PubMed: 7537730]

18. Christensen BM, Zelenina M, Aperia A, Nielsen S. Localization and regulation of PKAphosphorylated AQP2 in response to V(2)-receptor agonist/antagonist treatment. Am J Physiol Renal Physiol 2000;278(1):F29-F42. [PubMed: 10644653] 
A

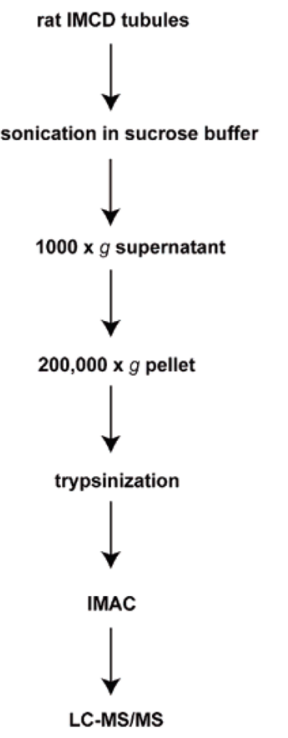

B

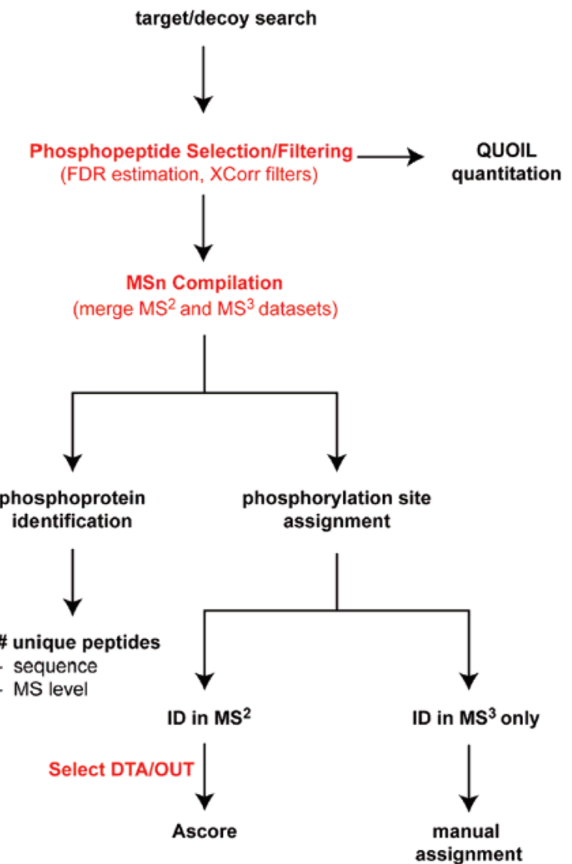

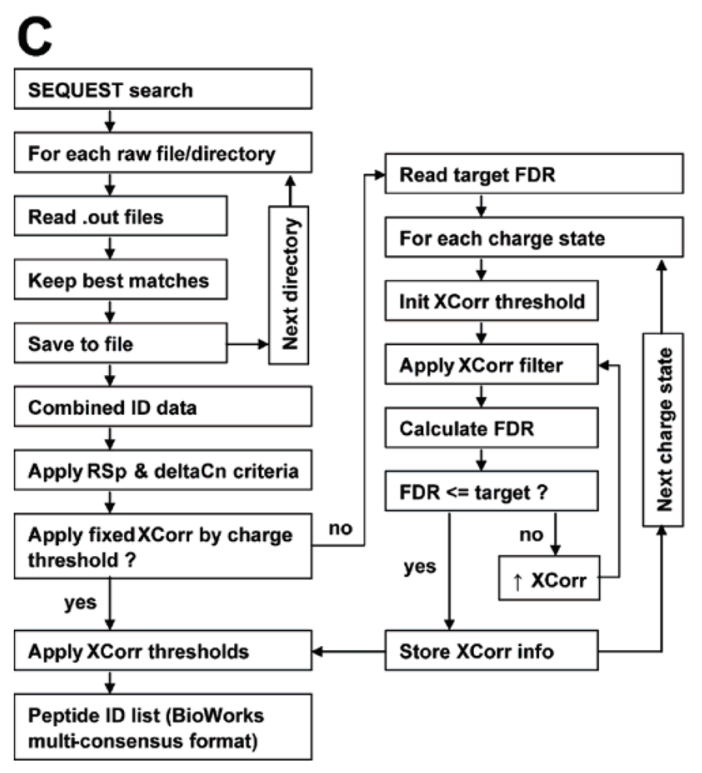

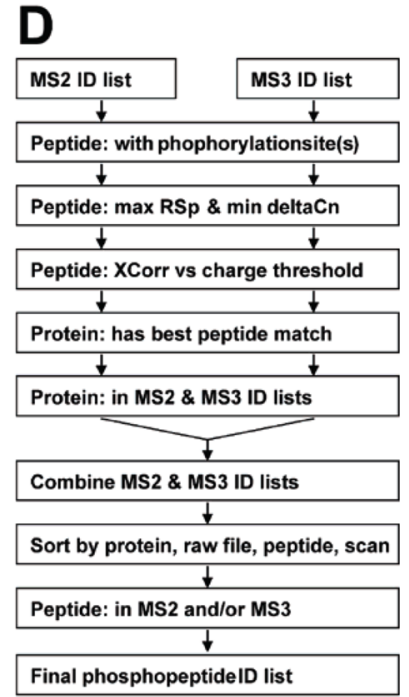

Figure 1.

Experimental approach. (A) IMCD cells were enriched from rat kidney inner medullas by enzymatic digestion followed by low-speed centrifugation. Cells were lysed by sonication in sucrose buffer. Differential centrifugation produced a $200000 \mathrm{~g}$ pellet which was digested with trypsin, followed by IMAC to enrich for phosphopeptides. Phosphopeptides were then analyzed by LC-MS/MS. (B) Postacquisition analysis. The 3 modules that comprise PhosphoPIC are indicated in red font. FDR $=$ false discovery rate; XCorr $=$ cross-correlation filter. (C) Detailed workflow for the "Phosphopeptide Selection/Filtering" module of PhosphoPIC. (D) Detailed workflow for the "MSn Compilation" module of PhosphoPIC. 
A

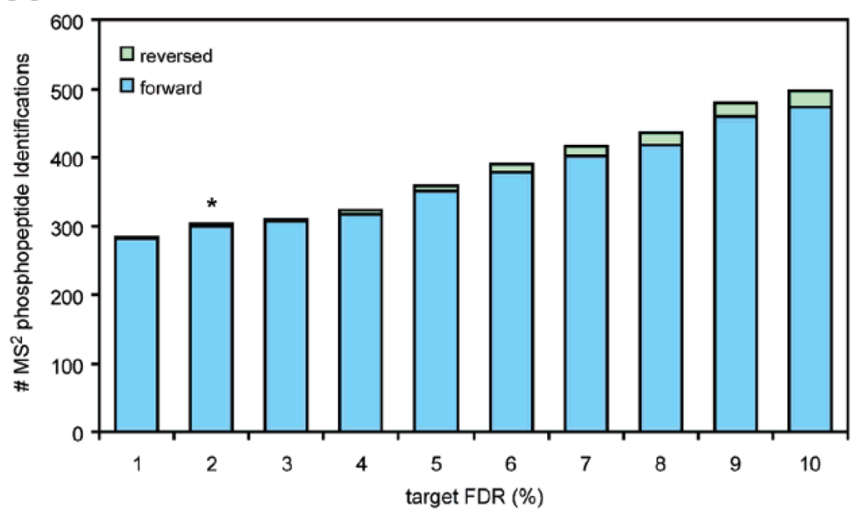

C

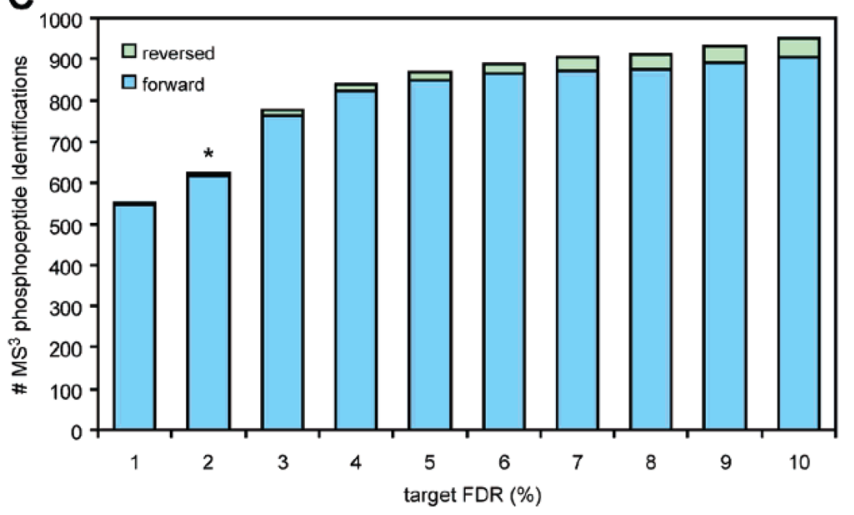

B

\begin{tabular}{|ccccc|}
\hline $\begin{array}{c}\text { forward } \\
\text { hits }\end{array}$ & $\begin{array}{c}\text { reversed } \\
\text { hits }\end{array}$ & $\begin{array}{c}\text { target } \\
\text { FDR (\%) }\end{array}$ & $\begin{array}{c}\text { actual } \\
\text { FDR (\%) }\end{array}$ & $\begin{array}{c}\text { adjusted } \\
\text { FDR (\%) }\end{array}$ \\
\hline 282 & 1 & 1.00 & 0.71 & 0.35 \\
300 & 2 & 2.00 & 1.32 & 0.67 \\
306 & 3 & 3.00 & 1.94 & 0.98 \\
317 & 6 & 4.00 & 3.72 & 1.89 \\
351 & 8 & 5.00 & 4.46 & 2.28 \\
379 & 11 & 6.00 & 5.64 & 2.90 \\
401 & 14 & 7.00 & 6.75 & 3.49 \\
418 & 17 & 8.00 & 7.82 & 4.07 \\
459 & 21 & 9.00 & 8.75 & 4.58 \\
473 & 24 & 10.00 & 9.66 & 5.07 \\
\hline
\end{tabular}

D

\begin{tabular}{|ccccc|}
\hline $\begin{array}{c}\text { forward } \\
\text { hits }\end{array}$ & $\begin{array}{c}\text { reversed } \\
\text { hits }\end{array}$ & $\begin{array}{c}\text { target } \\
\text { FDR }(\%)\end{array}$ & $\begin{array}{c}\text { actual } \\
\text { FDR }(\%)\end{array}$ & $\begin{array}{c}\text { adjusted } \\
\text { FDR (\%) }\end{array}$ \\
\hline 548 & 2 & 1.00 & 0.73 & 0.36 \\
618 & 5 & 2.00 & 1.61 & 0.81 \\
764 & 11 & 3.00 & 2.84 & 1.44 \\
821 & 16 & 4.00 & 3.82 & 1.95 \\
847 & 21 & 5.00 & 4.84 & 2.48 \\
864 & 25 & 6.00 & 5.62 & 2.89 \\
872 & 31 & 7.00 & 6.87 & 3.56 \\
875 & 35 & 8.00 & 7.69 & 4.00 \\
890 & 41 & 9.00 & 8.81 & 4.61 \\
904 & 45 & 10.00 & 9.48 & 4.98 \\
\hline
\end{tabular}

Figure 2.

Estimation of false discovery rates. Both $\mathrm{MS}^{2}(\mathrm{~A}, \mathrm{~B})$ and $\mathrm{MS}^{3}(\mathrm{C}, \mathrm{D})$ phosphopeptide datasets were filtered using target false discovery rates (FDR) from 1 to $10 \%$. The number of peptides identified from the forward database (blue) as well as from the reversed database (green) increases as the FDR increases owing to the application of less stringent XCorr filters. A target FDR of $2 \%(*)$, which corresponded to an actual FDR of $1.32 \%$ for $\mathrm{MS}^{2}$ and $1.61 \%$ for MS, 3 was used for subsequent phosphopeptide identification and site assignment. Adjusted FDR is calculated after removal of hits from the reversed database.

J Proteome Res. Author manuscript; available in PMC 2008 September 1. 


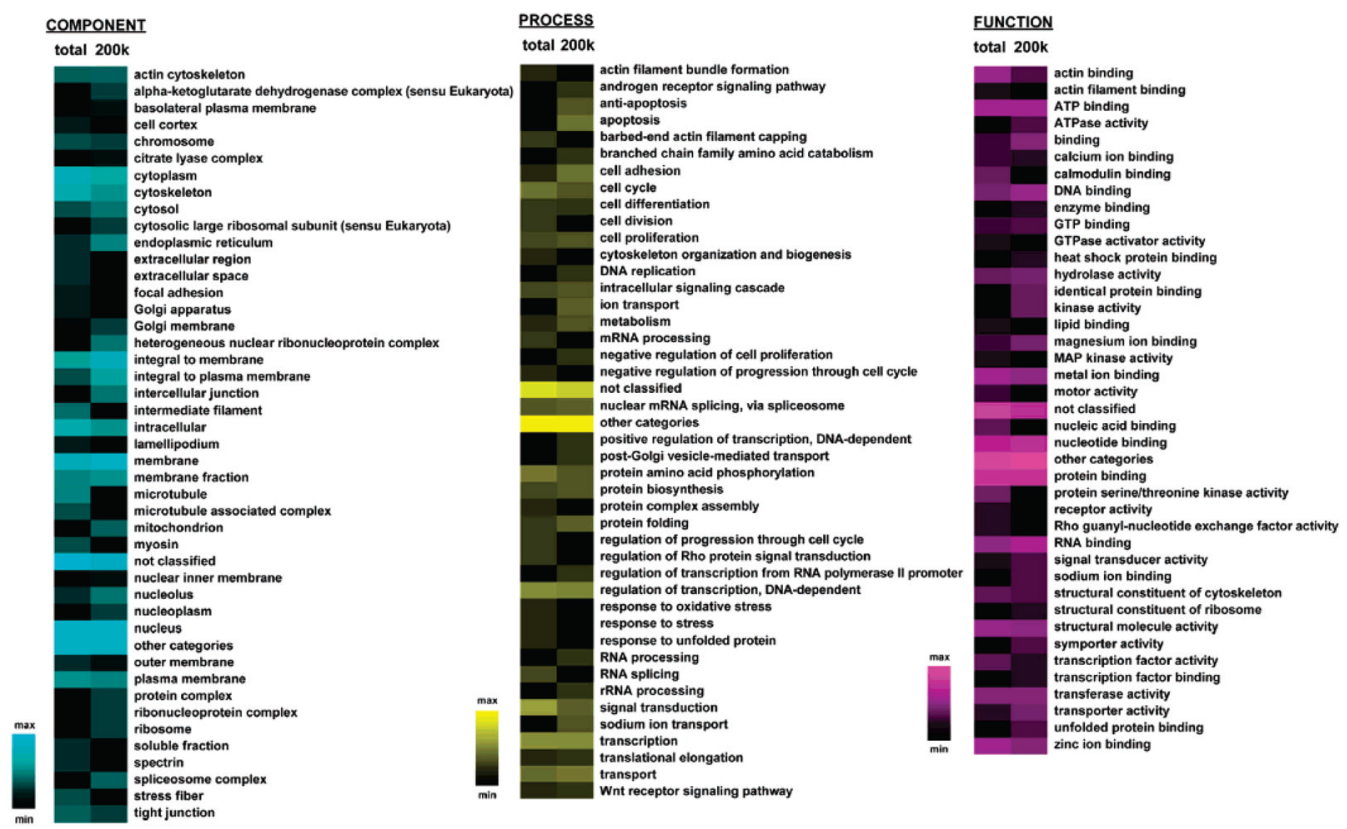

Figure 3.

Functional classification of identified IMCD phosphoproteins. Heat maps were constructed from the three major Gene Ontology (GO) categories: cellular component (cyan), biological process (yellow), and molecular function (magenta). The percentage of phosphoproteins associated with each individual GO term has been $\log _{2}$ transformed and then converted into a 16 -bit grayscale intensity value $(\min =0, \max =255)$. A direct comparison of whole cell lysate (total) with the membrane-enriched fraction (200k) indicates an increased number of phosphoproteins in the $200 \mathrm{k}$ fraction that have GO terms associated with membrane localization/membrane transport functions. 


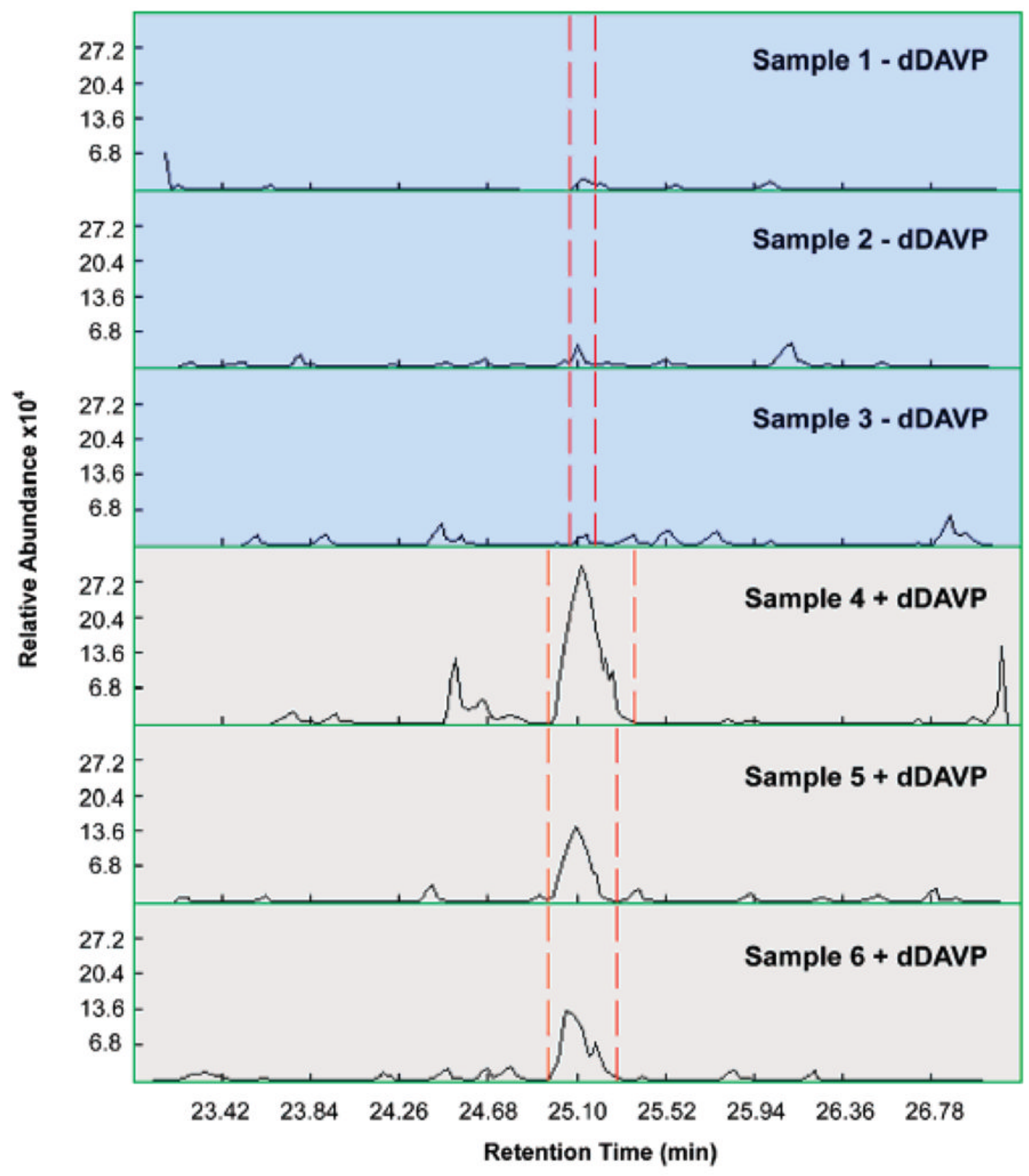

Figure 4.

Label-free quantification using QUOIL software. Phosphopeptides from IMCD samples incubated in the absence or presence of $10 \mathrm{nM}$ dDAVP for 10 min were selected using PhosphoPIC software and then quantified using a label-free approach based on normalized peak area ratios obtained from reconstructed ion chromatograms. Shown are the extracted ion chromatograms for a triply phosphorylated AQP2 peptide (RQpS-VELHpSPQpSLPR). The dashed red lines indicate the beginning and the end of the peaks that were quantified. The relative abundance of this AQP2 phosphopeptide was increased an average of 20.7-fold in samples that were incubated with $\operatorname{dDAVP}(n=3)$. 
Table 1

Summary of IMCD Phosphopeptide Data Analyzed by PhosphoPIC

\begin{tabular}{ll}
\hline Total phosphopeptides & 925 \\
Nonredundant phosphopeptides & 268 \\
False positives & 7 \\
Actual FDR & $1.5 \%$ \\
Adjusted FDR & $0.76 \%$ \\
Total proteins & 173 \\
Proteins in MS ${ }^{2}$ only & 22 \\
Proteins in $\mathrm{MS}^{3}$ only & 101 \\
Proteins in $\mathrm{MS}^{2}$ and MS & \\
Proteins unique to the membrane-enriched fraction of IMCD & 50 \\
\end{tabular}




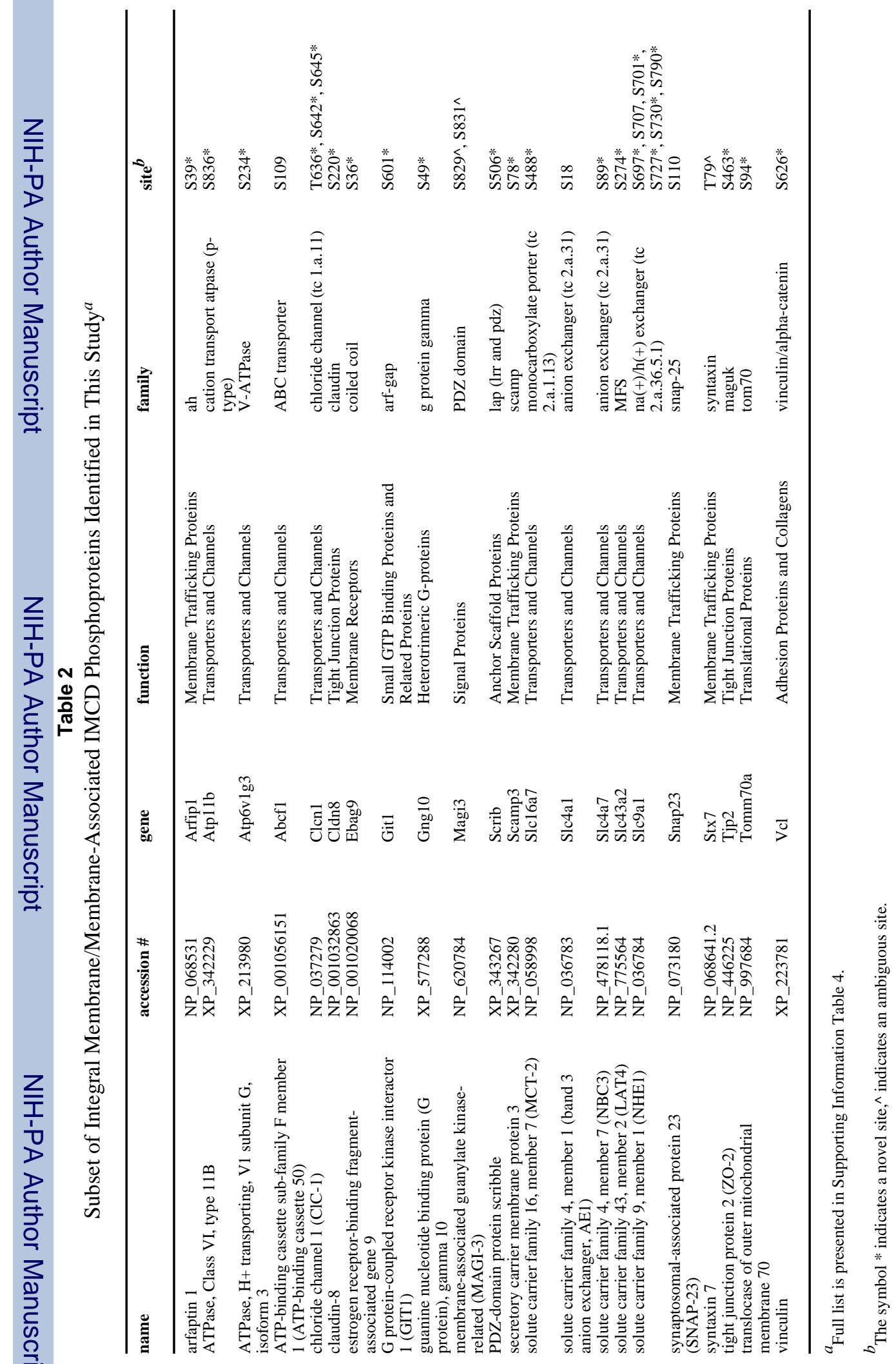

